\title{
Limitations of Different PCR Protocols Used in Diagnostic Laboratories: A Short Review
}

\author{
Masoud Hajia \\ Department of Molecular Biology, Health Reference Laboratory, Ministry of Health and Medical Education, Tehran, Iran \\ Received:25 March 2018;Accepted:11 April 2018;
}

ABSTRACT

Currently, different highly sensitive nucleic acid detection-based protocols used in medical diagnostic laboratories need specific working conditions, and if no optimization is performed, misleading results can be obtained. Limitations of each protocol is different in obtaining an appropriate result. Awareness of these limitations plays an important role in providing a reliable result for physicians and patients. The present study aims to investigate these limitations.

Keywords: Medical diagnostic laboratory, Molecular diagnosis

Corresponding Information: Dr. Masoud Hajia, Department of Molecular Biology, Health Reference Laboratory, Ministry of Health and Medical Education, Tehran,Iran. Email: massoudhajia@yahoo.com

Copyright (C) 2018. This is an open-access article distributed under the terms of the Creative Commons Attribution-noncommercial 4.0 International License which permits

\section{Introduction}

Polymerase Chain Reaction (PCR) technique has faced many developments since its inception. These developments not only have been used to improve the quality of materials and devices, but also the technique itself has undergone many modifications $(1,2)$. The developments have been towards quality improvement of the technique, increase of its sensitivity and specificity, and making it efficient in other laboratory areas, such as typing, cost-saving, higher speed in giving results ( 2 or 3 hours), and finally detection of a large number of samples at the same time (3).

This technique is based on using a pair of specific primers complementary to the target genome, Taq polymerase enzyme, and nucleotides (4), which are three main components of the reaction. Primers are oligonucleotide sequences, which by provide the possibility of genome synthesis through a series of repetitive cycles until the mass of amplified product can be detectable in different ways $(5,6)$.

One of the main characteristics of a test is its detection limit that is called "dynamic range". Detection limit or range is different in various PCR methods. Therefore, in line with quality improvement of the technique, detection limit of the test must be considered in addition to the parameters, such as reaction speed, cost-saving for each test, and good sensitivity and specificity. This limit is determined with minimum and maximum detection limits. The lowest detectable value of the analyte present in the sample and detectable in the test, is called "minimum detection limit" (8). To obtain the minimum detection limit, accurate optimization of the protocol and standardization of all involving factors, should be performed. In the absence of appropriate optimization, due to the interference of various factors, some of the samples containing low level of target genome may be reported as negative, which are actually 
false-negative. The number of reported false negatives in this condition depends on the lowest detection level of the test. Moreover, it should be noted that the conditions provided for the PCR reaction, support the amplification of a certain number of product. It should also be noted that the sensitivity of the test is completely different from detection level. The level of test sensitivity is basically defined by ability of the test in detecting different known types of an organism.

If a protocol is not able to identify come types, it have not sufficient sensitivity to identify the organism. In the case of no optimization, the protocol will not even be able to detect this number of types. According to this definition, detection limit of the test is used for detectable types. In the case of no optimization, the protocol cannot detect this number of types.

An appropriate sensitivity depends on the initial design of the protocol and selection of proper primer sequences, but the range of detection level is dependent on proper optimization of the amplification conditions. In addition to the effect of optimization on the detection level, improvement of product detection methods also has a significant impact on the process (9).

One of the main objectives of researchers in designing different types of protocols is to improve the detection level and the power of the test in detecting the lowest level of genome in samples $(10,11)$. In previous articles, we discussed the errors in samples and the abilities of different genomic purification methods (12). Given that this technique is implemented as different laboratory protocols (13), in this study, we aims to discuss advantages and limitations of these methods after explaining each procedures and to present a clear picture of their application.

\section{Different employed protocols}

Routine PCR method: This technique is a simple PCR method, which is performed the same as introduced about two decades ago, and the amplified product is evaluated by gel electrophoresis. In the case of appropriate amplification, the product mass that is at least 1-10 ng of genomic DNA, should be visible in a certain situation. Most of the kits available in the market are introduced this way due to their simple set up instructions.

This method has some special limitations and advantages. One of the most important limitations of this method is high risk of contamination, which takes longer time to give the result. In this method, amplicons can be easily spread over the environment. Hence, isolation of work environment from the external environment and creation of work disciplines are of great importance due to false positive results.

Although, in the early theory, the PCR's ability to detect target sequences was introduced to be less than 10 per sample, but in different types of protocols, researchers could rarely design a test that meets this detection level. Currently, the detection level of the method is much lower than the above-mentioned level, and its sensitivity has decreased due to the interference of various factors. Lack of use of a standard genomic purification method, using low-quality materials, and no optimization of the test, are some of the important influencing factors. However, test sensitivity will decrease with small changes in working conditions. Therefore, continuous quality control of used facilities should be taken into consideration (14).

In the case of using this method for detection, it is recommended that in addition to the use a secondary protocol as an internal control, the positive control be used at the minimum level capable of becoming positive. In this case, only the probability of contamination is reduced, and also the likelihood of observing low-quality extraction process and/or used materials, are determined.

PT-PCR technique: PCR technique has been principally designed to amplify a DNA segment. 
Therefore, in cases in which the detection target is cellular mRNA and/or RNA genome viruses, it is necessary that at first the RNA is converted to DNA (complementary DNA or cDNA), and then PCR reaction is performed.

In this method, at first, synthesis of cDNA is performed using a specific primer and/or a random hexamer primer and a reverse transcriptase enzyme. Then, the target genome was amplified in the PCR process. In addition to the limitations and advantages of the routine PCR method, the sensitivity of working with RNA imposes additional limitations on the experiment (15).

Multiplex PCR: In this method, several pairs of primer are used for different target molecules in the reaction mixture. Such a method can be theoretically used for simultaneous amplification of sequences of several pathogen microorganism in a reaction mixture (16). This method can be useful for clinical laboratories. Relative decrease in sensitivity is one of the limitations of this method in comparison with single PCR method. Using this method for the tests designed for human genome may pose no significant problem, but using it for detection of the infectious agents requires an accurate primer design and comprehensive optimization of the test (17).

Nested PCR: In this method, several amplification cycles are performed using a set of primers (18), and then the product of the amplification is reamplified using a set of primers internal to the sequence of the first product (19). The method is highly sensitive, but has some limitations. After performing the first stage, the products should be transferred to a new tube and/ or the materials of the second stage are added to it, which in both cases it is necessary to open the cap of the tube. Such limitations might be overcome through physical separation of the reaction mixture from the first and second sets of primers by creating an oil phase. After amplification by the first set of primers, the separated phases are mixed and their content is amplified by the second set of primers. The primers of the first stage can be genus- or species-specific. This method is used to differentiate different species and genotypes(19). If addition of the sample to reaction mixture in the second stage, is performed in a space out of the initial and electrophoresis space, the spread of amplicons and risk of contamination will decrease.

PCR hybridization method: This method is used to increase the detection level of the test and to improve its specificity. It is based on providing necessary conditions for hybridization of a specific probe to PCR product of the first stage (20). Since the probe is labeled, it can be easily detected. The risk of environmental contamination is high in this method, but its detection level is far better than the previous methods. In addition, by designing different probes, this method can be used as a genotyping method (21). To reduce the risk of contamination, all post-amplification procedures should be performed outside the initial space (22).

PCR ELISA method: This method has been integrated with ELISA method to increase the detection level of the test and to quantitatively determine the number of genomes in the sample. At first, the two PCR-produced DNA strands are separated from each other, hybridized with biotinlabelled probes, and transferred to streptavidincoated microtiter plates.

Specific affinity of streptavidin to biotin causes the strand-probe to bind to the wall of microtiter plates. Then, the result of the reaction can be observed and measured by enzymatic reactions, after adding the genome. Quantitative evaluation is one of the advantages of this method, while laborious and time-consuming are its major limitations (17).

Fluorescent Amplification-based Specific Hybridization method: This method is based on using a specific probe that is hybridized to the target strand at the same time as the primers. Two ends of the probe are labeled with a fluorophore (as a reporter) and a quencher. This probe normally form a loop due to the presence of 
complementary bases at its two ends $(23,24)$.

In the absence of complementary sequences in the target genome, this loop hinders the radiation, so there will be no measurable light. In the case of the presence of a specific target strand, the probe binds to it, but is degraded due to the 5 '-endonuclease activity of Taq-polymerase enzyme, and the fluorophore is released and can be quantitatively measured using a detector (25). Quencher is made from a material with a wavelength beyond the visible spectrum. The released labeled material can be measured in a certain wavelength according to the dye used in the reporter molecule (26). Among the limitations of this method, we can refer to unwanted false reactions occurring due to radiation of the labeled material that is released in different conditions (26).

Use of internal control: An internal control is used to confirm the actual results and the absence of potential inhibitors remaining from PCR process, so that the primer not only binds to the specific template, but also to a sequence that has been added to the kit. This sequence is composed of a plasmid, which is capable of producing a longer length product (27). In case of absence of specific DNA in the patient's purified sample, this region is amplified and produces a larger size product, which is different from the main product and detectable and distinguishable from the positive control (28). In the case of the presence of primer-specific DNA, it will be amplified more than the internal control due to its smaller size. A system similar to the main probe system can be used to detect the product of internal control, but type of the labeled molecule is different and measured at different wavelength than that of specific reaction probe (29).

High speed in giving results from the beginning of the test, no need for electrophoresis, and consequently no spread of amplicons in the environment, are some appropriate conditions occurrence of no false-positive reactions. Its limitation is need for a specific detector to measure the wavelength of the labeled molecule (29).

Developed rapid PCR method and real time PCR: PCR development process has been always towards increase in the reaction speed. In addition, use of novel detection methods reduce the risk of environmental contamination caused by spread of amplicons. In these systems, the two above-mentioned purposes have been fulfilled. In this method, new types of thermocyclers with specific detection system are used, and the reaction process can be monitored at any time through a computer screen. In the types that work based on using LightCycler, faster heat exchange can be done due to extremely low volume of the reaction mixture and use of capillary tubes. Therefore, the whole reaction takes less than half an hour, but such a method has its own subtleties and complications. In different real-time PCRs, the capillary tubes have been replaced with specific $0.2 \mathrm{ml}$ volume tubes. The required time for the whole reaction is slightly more than LightCycler types (30).

Reaction mechanism: In the designed protocols, the amount of product is quantitatively measured using labeled materials. Using different types of labels have made developments in the use of PCR. Different methods have been investigated and used for measuring the amount of the product by labeled materials, including SYBR Green dye and labeled probes like in PCR-hybridization mechanism and/or in FLASH method. Currently, in the designed kits, there is a trend to use the mechanism provided for FLASH technique. In the provided probes, different labeled molecules have been used as the reporter. In this system, the internal control is used with the same conditions designed for the main reaction. In these two reactions, measuring the amount of product at any time is possible using the genomic standards used through the process, and during the PCR process and formation of the product, we can monitor the increased level of fluorescence, which has been resulted from 
product accumulation (31).

Quantitative measurement of the product, significant reduction of contamination, increased speed of the procedure, and no need for the space required for post-PCR, are among the advantages of this method. Moreover, high cost of test performance and need for initial investment are some of its current important limitations.

\section{Conflict of interest}

The authors declare that there is no conflict of interests.

\section{References}

1. Van Gelder RN. Cme review: polymerase chain reaction diagnostics for posterior segment disease. Retina 2003;23(4):445-52.

2. Garibyan L, Avashia N. Polymerase chain reaction. J Invest Dermatol. 2013;133(3):e6.

3. Kafwabulula M, Ahmed K, Nagatake T, Gotoh J, Mitarai S, Oizumi K, et al. Evaluation of PCR-based methods for the diagnosis of tuberculosis by identification of mycobacterial DNA in urine samples. Int J Tuberc Lung Dis 2002;6(8):732-7.

4. Mullis KB. The unusual origin of the polymerase chain reaction. Sci Am 1990;262(4):56-61, 64-5.

5. National Laboratory of Enteric Pathogens BoMLCfDC. The polymerase chain reaction: An overview and development of diagnostic PCR protocols at the LCDC. Can J Infect Dis 1991;2(2):89-91.

6. Barken KB, Haagensen JA, Tolker-Nielsen T. Advances in nucleic acid-based diagnostics of bacterial infections. Clin Chim Acta 2007;384(1-2):1-11.

7. Yamamoto Y. PCR in diagnosis of infection: detection of bacteria in cerebrospinal fluids. Clin Diagn Lab Immunol. 2002;9(3):508-14.

8. Walker MJ, Montemagno C, Bryant JC, Ghiorse WC. Method detection limits of PCR and immunofluorescence assay for Cryptosporidium parvum in soil. Appl Environ Microbiol. 1998;64(6):2281-3.

9. Speers DJ. Clinical applications of molecular biology for infectious diseases. Clin Biochem Rev 2006;27(1):3951.
10. Dore GJ, Kaldor JM, McCaughan GW. Systematic review of role of polymerase chain reaction in defining infectiousness among people infected with hepatitis $\mathrm{C}$ virus. BMJ 1997;315(7104):333-7.

11. Weier HU, Gray JW. A programmable system to perform the polymerase chain reaction. DNA 1988;7(6):441-7.

12. Hajia M, Fallah F, Angoti G, Karimi A, Rahbar M, Gachkar L, et al. Comparison of Methods for Diagnosing Brucellosis. Lab Medicine 2013;44(1):29-33.

13. Hajia M, Safadel N, Samiee SM, Dahim P, Anjarani S, Nafisi N, et al. Quality assurance program for molecular medicine laboratories. Iran J Public Health 2013;42(Supple1):119-24.

14. Panteghini M, Forest JC. Standardization in laboratory medicine: new challenges. Clin Chim Acta 2005;355(1-2):1-12.

15. Overbergh L, Giulietti A, Valckx D, Decallonne $\mathrm{R}$, Bouillon R, Mathieu C. The use of real-time reverse transcriptase PCR for the quantification of cytokine gene expression. J Biomol Tech. 2003;14(1):33-43.

16. Hajia M, Rahbar M, Rahnami Farzami M, Dolatyar A, Imani M, Saburian R, et al. Efficacy of multiplex PCR procedure for Iranian Streptococcus pneumoniae isolates. Caspian J Intern Med 2014;5(2):109-13.

17. Shahrokhabadi R, Rahimi E, Mommtaz H, Poursahebi R, Doostmohamadi S. The Efficacy of Multiplex PCR in Comparison with Agglutination and ELISA in Diagnosis of Human Brucellosis. Zahedan $\mathrm{J}$ Research Med Sci 2014;16(4):24-8.

18. Stabel JR, Bannantine JP. Development of a nested PCR method targeting a unique multicopy element, ISMap02, for detection of Mycobacterium avium subsp. paratuberculosis in fecal samples. J Clin Microbiol 2005;43(9):4744-50.

19. Prakash P, Mishra OP, Singh AK, Gulati AK, Nath G. Evaluation of nested PCR in diagnosis of typhoid fever. J Clin Microbiol 2005;43(1):431-2.

20. Bentley RW, Leigh JA. Development of PCR-based hybridization protocol for identification of Streptococcal species. J Clin Microbiol 1995;33(5):1296301.

21. Adler M, Wacker R, Niemeyer CM. Sensitivity 
by combination: immuno-PCR and related technologies. Analyst 2008;133(6):702-18.

22. Rossi MC, Gori A, Zehender G, Marchetti G, Ferrario G, De Maddalena C, et al. A PCR-colorimetric microwell plate hybridization assay for detection of Mycobacterium tuberculosis and M. avium from culture samples and Ziehl-Neelsen-positive smears. J Clin Microbiol 2000;38(5):1772-6.

23. Smith CJ, Osborn AM. Advantages and limitations of quantitative PCR (Q-PCR)-based approaches in microbial ecology. FEMS Microbiol Ecol 2009;67(1):620.

24. Khatami F, Heidari M, Khatami M. Rapid Detection of Escherichia coli O157: H7 by Fluorescent Amplification-Based Specific Hybridization (FLASH) PCR. Iran Red Crescent Med J 2012;14(9):594-8.

25. Postollec F, Falentin H, Pavan S, Combrisson J, Sohier D. Recent advances in quantitative PCR (qPCR) applications in food microbiology. Food Microbiol 2011;28(5):848-61.

26. McKay JA, Murray GI, Keith WN, McLeod HL. Amplification of fluorescent in situ hybridisation signals in formalin fixed paraffin wax embedded sections of colon tumour using biotinylated tyramide. Mol Pathol 1997;50(6):322-5.
27. Herwegh S, Carnoy C, Wallet F, Loiez C, Courcol RJ. Development and use of an internal positive control for detection of Bordetella pertussis by PCR. J Clin Microbiol 2005;43(5):2462-4.

28. Eisler DL, McNabb A, Jorgensen DR, IsaacRenton JL. Use of an internal positive control in a multiplex reverse transcription-PCR to detect West Nile virus RNA in mosquito pools. J Clin Microbiol 2004;42(2):841-3.

29. Rosenstraus M, Wang Z, Chang SY, DeBonville D, Spadoro JP. An internal control for routine diagnostic PCR: design, properties, and effect on clinical performance. J Clin Microbiol 1998;36(1):191-7.

30. Gaydos CA. Review of use of a new rapid real-time PCR, the Cepheid GeneXpert(R) (Xpert) CT/ NG assay, for Chlamydia trachomatis and Neisseria gonorrhoeae: results for patients while in a clinical setting. Expert Rev Mol Diagn 2014;14(2):135-7.

31. Imani Fooladi AA, Tarvedi Zadeh Y, Mehrab R, Halabian R, Azizi T. Evaluation of FLASH - PCR forrapid detection of Mycobacterium tuberculosis from clinical specimens. Iran J Microbiol 2013;5(4):383-90.

\section{How to cite this article}

Hajia M. Limitations of Different PCR Protocols Used in Diagnostic Laboratories: A Short Review. Mod Med Lab J. 2018;1(1):1-6 